\section{Spritzen-Schmerz: Einfach wegschauen!}

Beim Erhalt einer Spritze einfach wegzusehen ist sinnvoll, wie eine deutsche Studie zeigt. Die Teilnehmer empfanden elektrische Schmerzreize an der eigenen Hand intensiver, wenn sie zusätzlich sahen, wie eine virtuelle Hand gepikst wurde. Auch die Berührung mit einem Q-Tip wurde bei gleichzeitigem Spritzen der virtuellen Hand mit messbaren Schmerzen verbunden. Die Erwartungshaltung spielt für die gemessene Schmerzintensität eine große Rolle: So fiel der Schmerz auch höher aus, wenn die Teilnehmer auf Spritze und den damit verbundenen Schmerz vorbereitet wurden.

Höfle M et al, Pain 2012, 153:1074-1081

\section{Kalziumtabletten: Herzinfarkt riskieren?}

Die regelmäßige Einnahme von Kalzium zur Vorbeugung einer Osteoporose erhöht möglicherweise das Herzinfarktrisiko. Das ergab die Auswertung der Daten von 23.980 Probanden einer prospektiven Studie. Das Herzinfarktrisiko stieg bei Kalziumeinnahme um 86\%. Auf die Häufigkeit von Schlaganfällen oder die kardiovaskuläre Sterblichkeit nahm das Kalzium keinen Einfluss. Das Ergebnis stellt die häufig unkritische Empfehlung der Nahrungsergänzung infrage, denn eine ausgewogene Ernährung reicht als Kalziumlieferant aus.

Li K et al, Heart 2012, 98:920-925

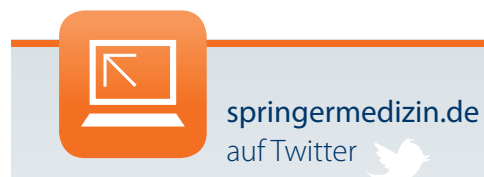

Auch zum Sommeranfang heißt es: „Lauschen“ Sie unserem Zwitschern und werden Sie zum „Follower"! > twitter.com/springermedizin
Kalk ist Kalk

\section{Harnsteine erhöhen Apoplex-Risiko}

Harnsteine sind ein Hinweis auf ein erhöhtes Schlaganfallrisiko, ganz egal ob die Konkremente in der Niere oder im Harnleiter sitzen.

Taiwanesische Forscher fanden eine eindeutige Assoziation zwischen Nierensteinen und dem Schlaganfallrisko. Demnach sind Harnsteinpatienten 1,43mal mehr gefährdet als Menschen ohne Konkremente, innerhalb von fünf Jahren einen Insult zu erleiden, unabhängig von Alter, Geschlecht oder Komorbiditäten. An der Studie nahmen über 25.000 Patienten mit neu diagnostizierten Harn- steinen teil, deren Daten mit mehr als 125.000 steinfreien Kontrollpersonen verglichen wurde. Bei den Steinträgern wurden pro 100 Personenjahre 1,78 Schlaganfälle registriert, in der Kontrollgruppe 1,25. Die Steinpatienten hatten deutlich häufiger Hochdruck, Diabetes, eine KHK, Herzinsuffizienz oder erhöhte Blutfettwerte. Eine solche Stoffwechsellage könne über die Arterienverkalkung auch zu dem erhöhten Schlaganfallrisiko beitragen. Der gemeinsame Mechanismus sei möglicherweise ein Mangel an Inhibitoren, die die Kalkbildung verhindern. (eo) Chung S-D et al, BJUI 2012 (online first)

\section{Hormonelle Kontrazeptiva im Vergleich „Pille zum Kleben“ fördert Thrombosen}

Frauen, die mit Hormonpflastern verhüten, haben ein achtmal so hohes Thromboserisiko wie Frauen, die keine hormonellen Kontrazeptiva einsetzen.

In einer dänischen Registerstudie mit gut 1,5 Mio. Frauen wurde das Thromboserisiko hormoneller Kontrazeptiva verglichen. Bei Frauen, die nicht hormonell verhüteten, traten hochgerechnet auf 10.000 Jahre 2,1 Thrombosen auf. Dagegen waren es beim Hormonpflaster 9,7, beim Vaginalring 7,8 und bei der Pille 6,2 Thrombosen. Die Wirkstoffe der „Pille zum Kleben“ sind Norelgestromin und Ethinylestradiol. Frühere Studien zeigten, dass der Östrogenspiegel bei Hormonpflastern bis zu $60 \%$ höher ist als mit der Pille. Der Vaginalring enthält neben Ethinylestradiol das
Drittgenerations-Gestagen Etonogestrel. Bei Anwendung von Vaginalringen erhöht sich die Konzentration des Transportproteins (SHBG) für die Sexualhormone Östrogen und Testosteron um das Fünf- bis Sechsfache. SHBG gilt als Marker für ein erhöhtes Thromboserisiko.

\section{Risiko mehr als verdoppelt}

Gegenüber der „Pille“war das Thromboserisiko mit dem Pflaster 2,3-fach erhöht, mit Vaginalringen in etwa verdoppelt. Subkutane Hormonimplantate ließen das Risiko nur leicht, Intrauterinpessare mit Levonorgestrel dagegen gar nicht steigen. Bei der Spirale ist vielleicht sogar mit einer Schutzwirkung zu rechnen. (eo) 